Article

\title{
Increased Risk of Multiple Outpatient Surgeries in African-American Carriers of Transthyretin Val122Ile Mutation Is Modulated by Non-Coding Variants
}

\author{
Renato Polimanti ${ }^{1, * \mathbb{D}}$, Yaira Z. Nuñez ${ }^{1}$ and Joel Gelernter ${ }^{1,2} \mathbb{D}$ \\ 1 Department of Psychiatry, Yale University School of Medicine and VA CT Healthcare Center, West Haven, \\ CT 06516, USA; yari.nunez@yale.edu (Y.Z.N.); joel.gelernter@yale.edu (J.G.) \\ 2 Departments of Genetics and Neuroscience, Yale University School of Medicine, New Haven, CT 06510, USA \\ * Correspondence: renato.polimanti@yale.edu; Tel.: +1-(203)-932-5711 (ext. 5745); Fax: +1-(203)-937-3897
}

Received: 23 January 2019; Accepted: 18 February 2019; Published: 22 February 2019

check for updates

\begin{abstract}
Background: African-Americans (AAs) have a 3.5\% carrier prevalence of Transthyretin (TTR) Val122Ile mutation (rs76992529), which is the genetic cause of a hereditary form of amyloidosis. Methods: We investigated the medical history of Val122Ile carriers and assessed the role of a non-coding variation in 4361 unrelated AAs. Results: We observed that the Ile122 allele was associated with a 6.8-fold increase in the odds of having 10 or more outpatient surgeries $\left(p=7.81 \times 10^{-5}\right)$. Stratifying the analysis by sex, the Ile122 allele was associated with a 15.2-fold increase in the odds of having 10 or more outpatient surgeries in men $\left(p=6.49 \times 10^{-7}\right)$. A similar sex difference was observed with respect to the association of Val122Ile with musculoskeletal and connective-tissue disorders in an independent cohort of British subjects $\left(n=361,194, p=2.47 \times 10^{-13}\right.$; $\left.n_{\text {male }}=167,020, p_{\text {male }}=4.02 \times 10^{-24}\right)$. In Val122Ile African-American carriers, we observed that haplotypes in the upstream region regulating TTR hepatic expression are associated with having 10 or more outpatient surgeries $\left(p=2.56 \times 10^{-9}\right)$. Conclusions: TTR Val122Ile showed a large effect with respect to an extreme phenotype identified in medical history that may be related to osteoarthritis, an early sign of the disease. Additionally, the non-coding variation appears to accelerate the negative consequences associated with Val122Ile mutation via TTR expression regulation.
\end{abstract}

Keywords: TTR amyloidosis; African ancestry; phenome-wide; V122I; medical history

\section{Introduction}

Transthyretin (TTR) amyloidosis is a life-threatening disease (Online Mendelian Inheritance in Man: \#105210) caused by coding mutations in the TTR gene. The disease is characterized by a wide range of clinical signs and symptoms, including peripheral neuropathy (sensory and motor), cardiomyopathy, autonomic neuropathy, gastrointestinal impairment, nephropathy, and ocular deposition [1,2]. Although more than 100 amyloidogenic variants have been identified in TTR, two mutations account for most of the cases worldwide, i.e., Val30Met (rs28933979) and Val122Ile (rs76992529) [3,4]. While Val30Met is mainly present in European and Japanese populations [5], V122Ile shows high frequency in individuals of African descent. In African Americans (AAs), the Ile122 allele frequency is $1.5 \%$, which corresponds to $3.5 \%$ of carriers [6,7]. The main disease phenotype associated with this mutation is restrictive amyloid cardiomyopathy, and it has been reported that homozygous individuals can be symptomatic in their late 50s and early 60s, while heterozygotes can develop congestive heart failure between the ages of 60 and 80 [8]. However, the actual extent of Val122Ile penetrance is still debated because of the differences observed between general-population cohorts and case-control studies [4]. Penetrance variability has also been observed for other TTR 
mutations, and previous studies suggested that genetic and environmental factors play a role in the phenotypic expression [9-12]. In particular, recent investigations observed that non-coding variation of the TTR gene may play a role in the complex genotype-phenotype correlation due to its effect on gene expression regulation $[13,14]$. Although no analysis has been conducted previously to test this hypothesis on Val122Ile AA carriers, an in silico study based on the 1000 Genomes Project reference panel observed that some Ile122 haplotypes present different non-coding structures, including multiple regulatory variants [10]. The observation that the known risk alleles occur on different haplotypic backgrounds with different regulatory variants supports the claim that the non-coding variation may modulate the phenotypic presentation among Val122Ile carriers.

The aim of the present study was to evaluate two hypotheses: (i) Val122Ile affects the medical history of carriers earlier than previously reported; (ii) Non-coding regulatory variation modulates the effects of Val122Ile among carriers. To test these hypotheses, we investigated a relatively young cohort (interquartile age range of 35 to 48 years) including 4361 unrelated AAs with 152 Val122lle carriers (150 Val122/Ile122 heterozygotes and two Ile122/Ile122 homozygotes). The initial analysis tested whether there were differences in the medical history (health status, primary diagnoses, hospitalizations, outpatient surgeries, emergency care events, and prescribed medications) between carriers and non-carriers. Then, we tested whether the haplotypic structure around the TTR gene in Val122Ile carriers affects the associations identified in the initial analysis.

\section{Methods}

\subsection{Study Population}

The sample was selected from the Yale-Penn cohort, which includes more than 14,000 participants. Subjects were recruited at five US clinical sites: Yale University School of Medicine (New Haven, CT, USA), the University of Connecticut Health Center (Farmington, CT, USA), the University of Pennsylvania Perelman School of Medicine (Philadelphia, PA, USA), the Medical University of South Carolina (Charleston, SC, USA), and McLean Hospital (Belmont, MA, USA). The institutional review board at each participating site approved the Yale-Penn project, which also extends to the present study, and written informed consent was obtained from each participant. Phenotypic information was collected using the SSADDA (Semi-Structured Assessment for Drug Dependence and Alcoholism) $[15,16]$. Biological samples for genetic analyses were collected from each subject at the time of the assessment. Data from the Yale-Penn cohort were mainly used to conduct genetic studies of psychiatric disorders and behavioral traits $[17,18]$. In the present study, we investigated 4361 unrelated individuals of African descent. As previously reported [19], ancestry and relatedness were verified using genetic information. Lifetime data regarding physical health were extracted from the medical history section of the SSADDA, which includes self-reported information regarding overall health status, primary diagnoses made by a doctor, hospitalizations, outpatient surgeries, emergency room visits, and the use of prescribed medications (Table 1).

Table 1. Characteristics of the study population. IQR: Interquartile range.

\begin{tabular}{lcc}
\hline & Study Population $(n=4361)$ & \\
Age years, IQR & & $35-48$ \\
Sex, Women $(\%)$ & $1924(44)$ \\
Val122Ile, Y $(\%)$ & $152(3.5)$ \\
& Health Status & \\
Excellent $(\%)$ & $675(15)$ \\
Very Good $(\%)$ & $1135(26)$ \\
Good (\%) & $1420(33)$ \\
Fair $(\%)$ & $940(22)$ \\
Poor $(\%)$ & $191(4)$ \\
\hline
\end{tabular}


Table 1. Cont.

\begin{tabular}{|c|c|}
\hline \multicolumn{2}{|l|}{ Doctor's diagnoses } \\
\hline High blood pressure, Y $(\%)$ & $1325(30)$ \\
\hline Migraine headaches, Y (\%) & $605(14)$ \\
\hline Brain injury or concussion, $\mathrm{Y}(\%)$ & $388(9)$ \\
\hline Been unconscious for longer than $5 \mathrm{~min}, \mathrm{Y}(\%)$ & $554(13)$ \\
\hline Epilepsy or have had a seizure, Y (\%) & $213(5)$ \\
\hline Meningitis or encephalitis, Y (\%) & $57(1)$ \\
\hline Stroke, Y (\%) & $88(2)$ \\
\hline Heart disease, Y (\%) & $182(4)$ \\
\hline Liver disease, Y (\%) & $481(11)$ \\
\hline Thyroid disease, Y (\%) & $134(3)$ \\
\hline Asthma, Y (\%) & $928(21)$ \\
\hline Diabetes, Y (\%) & $374(9)$ \\
\hline Cancer, Y (\%) & $108(2)$ \\
\hline \multicolumn{2}{|l|}{ Hospitalizations } \\
\hline $\mathrm{N}, \mathrm{IQR}$ & $0-3$ \\
\hline$\geq 10, \mathrm{Y}(\%)$ & $161(4)$ \\
\hline \multicolumn{2}{|l|}{ Outpatient surgeries } \\
\hline$>0, Y(\%)$ & $1945(45)$ \\
\hline$\geq 10, Y(\%)$ & $24(0.6)$ \\
\hline \multicolumn{2}{|l|}{ Emergency room visits } \\
\hline N, IQR & $0-3$ \\
\hline$\geq 10, Y(\%)$ & $300(7)$ \\
\hline \multicolumn{2}{|l|}{ Prescription medications } \\
\hline for nerves, $\mathrm{Y}(\%)$ & $610(14)$ \\
\hline for sleep, Y (\%) & $924(21)$ \\
\hline for depression, $\mathrm{Y}(\%)$ & $1012(23)$ \\
\hline for headaches, Y (\%) & $292(7)$ \\
\hline for energy, $Y(\%)$ & $95(2)$ \\
\hline containing steroids, Y (\%) & $451(10)$ \\
\hline
\end{tabular}

\subsection{Genetic Data}

Biological samples for DNA extraction collected at the time of the phenotypic assessment were used to conduct genome-wide array-based genotyping. The 4361 individuals included in the present analyses were genotyped with two different genome-wide arrays: 2763 using the Illumina HumanOmni1-Quad v1.0 microarray, and 1598 using the Illumina HumanCoreExome array. Details of the genotyping procedures were described previously [20]. Val122Ile (rs76992529) was included among the markers on the Illumina HumanCoreExome array. Val122Ile genotype for the subjects analyzed with the Illumina HumanOmni1-Quad v1.0 microarray was imputed using the Michigan Imputation Server [21] and the 1000 Genomes Project Phase 3 Reference Panel [22]. We obtained high-quality imputation of the Val122Ile mutation with an INFO score $=0.98$. The reliability of the imputation of the Val122Ile mutation was confirmed by the fact that we observed a perfect concordance between genotype data from the Illumina HumanCoreExome array and imputation data from the Illumina HumanOmni1-Quad v1.0 microarray in 89 individuals (3 Val122Ile carriers and 86 non-carriers) genotyped with both arrays. Haplotype association analysis in Val122Ile carriers was conducted using imputed data obtained from both genome-wide arrays. We used high-quality imputed variants (INFO score $>0.95$, minor allele frequency $>5 \%$; Table S1) in a $2 \mathrm{Mb}$ region that included the upstream region, TTR coding and non-coding sequences, and the downstream region (GRCh37/hg19 chr 18: $28,171,770-30,174,635)$.

\subsection{Statistical Analysis}

Plink 1.9 [23] was used to implement logistic, and linear regression analyses to calculate the association between Val122Ile and phenotypic traits (binary and quantitative, respectively) considering 
an additive genetic model. Quantitative traits were normalized using appropriate Box-Cox power transformations before being entered into the analysis. Quantitative traits with non-normal distribution after Box-Cox transformation were converted to binary variables using different thresholds. Age, sex, and 10 ancestry principal components were included as covariates in the regression models. For Val122Ile association analysis, we applied a Bonferroni-based threshold for multiple testing correction accounting for the number of phenotypes tested $\left(n=26, p=1.92 \times 10^{-4}\right)$. Haploview 4.2 [24] was used to conduct the haplotype association analysis considering V122I carriers only. Linkage disequilibrium (LD) blocks were defined considering the algorithm proposed by Gabriel et al. [25]. Bonferroni correction for the haplotype association analysis accounted for the number of haplotypes tested $\left(n=590, p=8.47 \times 10^{-5}\right)$. Functional annotation analyses were conducted using Haploreg v4.1 [26] and RegulomeDB [27]. The GTEx v7 [28] data were used to investigate how non-coding variation affects TTR gene expression in the liver, which is the main source of the TTR protein.

\section{Results}

Table 1 reports the characteristics of the sample investigated, including information regarding demographics, Val122Ile frequency, and the traits investigated in the association analysis. Among 4361 individuals, we identified 152 Val122Ile carriers (3.48\%) with 150 Val122/Ile122 heterozygotes and 2 Ile122/Ile122 homozygotes. No Val122Ile carriers were identified in the Yale-Penn participants of European descent. Figure 1 shows the results of Val122Ile association analysis with respect to the tested traits related to medical history. Details of the association results are reported in Table S2.

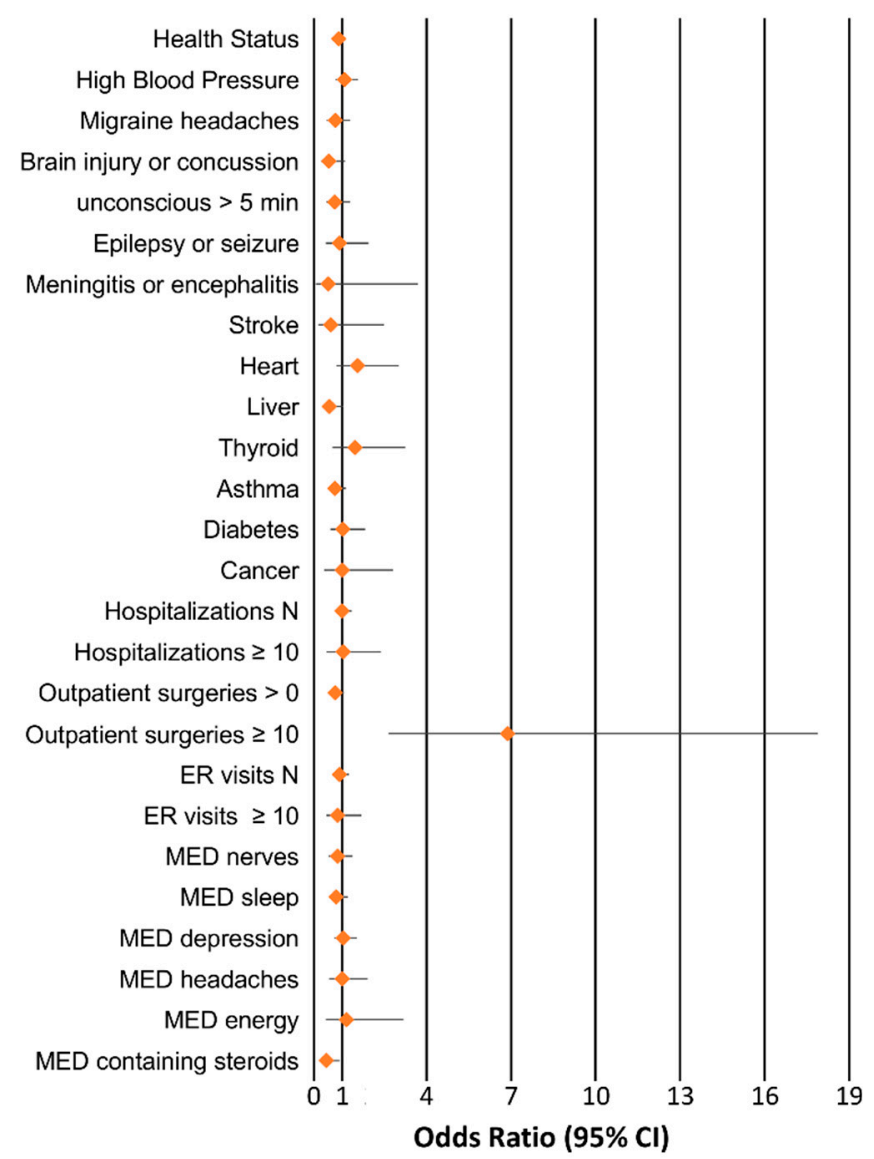

Figure 1. Associations between Transthyretin (TTR) V122Ile and traits related to medical history. Odds ratio and $95 \%$ confidence intervals are reported for each association.

We observed a significant association of Val122Ile that survived Bonferroni multiple testing correction: the I122 allele was associated with a 6.9-fold increase in the odds of having 10 or more 
outpatient surgeries, Odds Ratio $(\mathrm{OR})=6.87, p=7.81 \times 10^{-5}$ (2.6\% carriers versus $0.5 \%$ non-carriers). Since there is previous evidence of high penetrance of the Val122Ile mutation in male carriers [29], we conducted a sex-stratified analysis for the phenotype identified. In male subjects (carriers $n=80$, non-carriers $n=2357$ ), the I122 allele was associated with a 15.2-fold increase in the odds of having 10 or more outpatient surgeries ( $p=6.49 \times 10^{-7}, 5.1 \%$ carriers versus $0.3 \%$ non-carriers). In female sample (carriers $n=72$, non-carriers $n=1852$ ), no Val122Ile carriers had more than 10 outpatient surgeries ( $0 \%$ carriers versus $0.6 \%$ non-carriers). No age effect was observed in genetic association in the overall cohort and in the male sample $(p>0.05)$. Since the trait identified is an extreme phenotype observed in a limited number of individuals $(n=24)$, we conducted a permutation analysis to verify whether the significant association might be inflated by the number of cases. We performed 10,000 permutations of the TTR Val122Ile genotypes with respect to the "having 10 or more outpatient surgeries" phenotype and verified that the finding observed was significantly different from the null distribution of the results obtained from the permuted datasets considering the whole cohort and the male-only sample (whole-cohort $p_{\text {permutation }}=4 \times 10^{-4}$, male-only $p_{\text {permutation }}<10^{-4}$, Figure 2 ). Among the permuted results, the most likely random scenario is that no case was a carrier of the Val122Ile mutation (whole cohort 4422/10,000; male-only sample 7575/10,000). In line with its clinical significance, TTR Val122Ile showed a large effect with respect to this extreme phenotype.
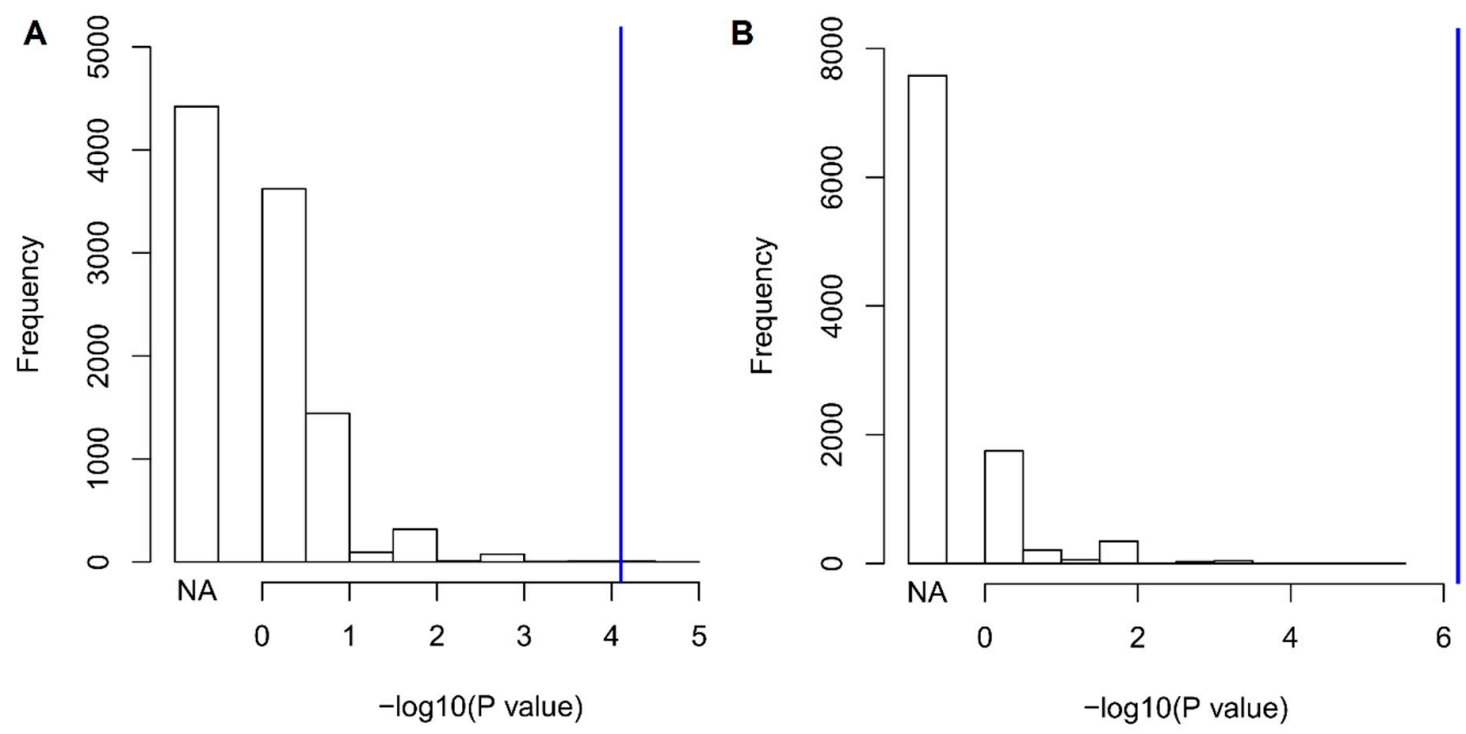

Figure 2. Null distribution of association results generated through 10,000 random permutations of having 10 or more outpatient surgeries with respect to Val122Ile genotype status in the whole cohort (A) and in the male-only sample (B). Blue lines indicate the observed values. The "NA" column represents the permutation results where no case is a carrier of the Val122Ile mutation.

Although the association was not significant $(p>0.05)$, Val122Ile was positively associated with heart disease $(\mathrm{OR}=1.55)$, and this association was slightly stronger in men than in women $\left(\mathrm{OR}_{\mathrm{men}}=\right.$ $\left.1.65 ; \mathrm{OR}_{\text {women }}=1.39\right)$. To verify whether the V122Ile mutation affects the relationship between the numbers of outpatient surgeries and the diagnosis of a heart disease, we tested the association between these health-related traits in carriers and non-carriers. Among V122Ile carriers, having 10 or more outpatient surgeries was associated with a 17.5-fold increase in the odds of having a diagnosis of heart disease $(p=0.022)$. A similar association strength was observed considering the male sample only (OR $=18, p=0.027$ ). Among non-carriers, there was no association between having 10 or more outpatient surgeries and the diagnosis of a heart disease $(p=0.836)$.

To verify whether non-coding regulatory variation modulates the association between Val122Ile and having 10 or more outpatient surgeries, we conducted a haplotype association analysis in Val122Ile carriers only. We analyzed a $2 \mathrm{Mb}$ region (GRCh37/hg19 chr 18: 28,171,770-30,174,635; upstream 
region, TTR gene, and downstream region), identifying 111 LD blocks that presented a total of 590 haplotypes detected (Table S3). Five haplotypes showed positive associations (i.e., higher frequency in cases than in controls) that survived Bonferroni multiple testing correction (Table 2, $p<8.45 \times 10^{-5}$ ). Table S4 reports the association results of all haplotypes tested.

Table 2. Haplotype associations with "having 10 or more outpatient surgeries" that survived multiple testing correction $\left(p<8.45 \times 10^{-5}\right)$ in the carriers-only sample $(n=152)$.

\begin{tabular}{cccccrc}
\hline $\begin{array}{c}\text { LD } \\
\text { Block }\end{array}$ & \multirow{2}{*}{ Location (Chr18) } & TTR Region & Haplotype & $\begin{array}{c}\text { Case-Control } \\
\text { Frequencies }\end{array}$ & Chi Square & $p$ Value \\
\hline$\# 12$ & $28,387,576-28,391,602$ & Upstream & GCACCGTATGGAGGGACCTC & $0.375,0.030$ & 24.395 & $7.85 \times 10^{-7}$ \\
$\# 14$ & $28,456,564-28,481,528$ & Upstream & TCTGTGTAGGGCC & $0.250,0.007$ & 35.494 & $2.56 \times 10^{-9}$ \\
$\# 15$ & $28,481,625-28,484,316$ & Upstream & GACAGA & $0.250,0.007$ & 35.494 & $2.56 \times 10^{-9}$ \\
$\# 16$ & $28,509,237-28,525,062$ & Upstream & TTTGTCCCTACAGGCCTTT & $0.500,0.061$ & 22.381 & $2.24 \times 10^{-6}$ \\
$\# 23$ & $28,629,162-28,640,811$ & Upstream & GAACTAATACCGA & $0.250,0.007$ & 35.344 & $2.76 \times 10^{-9}$ \\
\hline
\end{tabular}

The significantly associated haplotypes are located in four LD blocks close to each other that cover a $253 \mathrm{~kb}$ region located $531 \mathrm{~kb}$ upstream of the TTR gene (Figure S1). Annotation analyses showed that variants included in these LD blocks present multiple signs of evidence of regulatory function including highly conserved sequences, enhancer histone marks, DNase hypersensitivity sites, protein binding sites, and transcription factor motif changes (Table S5). Among the variants present in the associated haplotypes, rs 9957088 showed the highest functionality score (RegulomeDB score $=3 \mathrm{a})$ and was significantly associated with TTR gene expression in the liver ( $\mathrm{C}$ allele: beta $=0.15$, $p=0.013$, Figure 3). Additionally, we verified protein changes associated with this variant in a large cohort of healthy blood donors $(n=3301)$ [30]. Although TTR was not among the proteins tested, rs9957088 showed the strongest association with haptoglobin protein levels ( $C$ allele: beta $=0.109$, $\left.p=1.02 \times 10^{-4}\right)$, with an effect direction concordant with TTR gene expression.

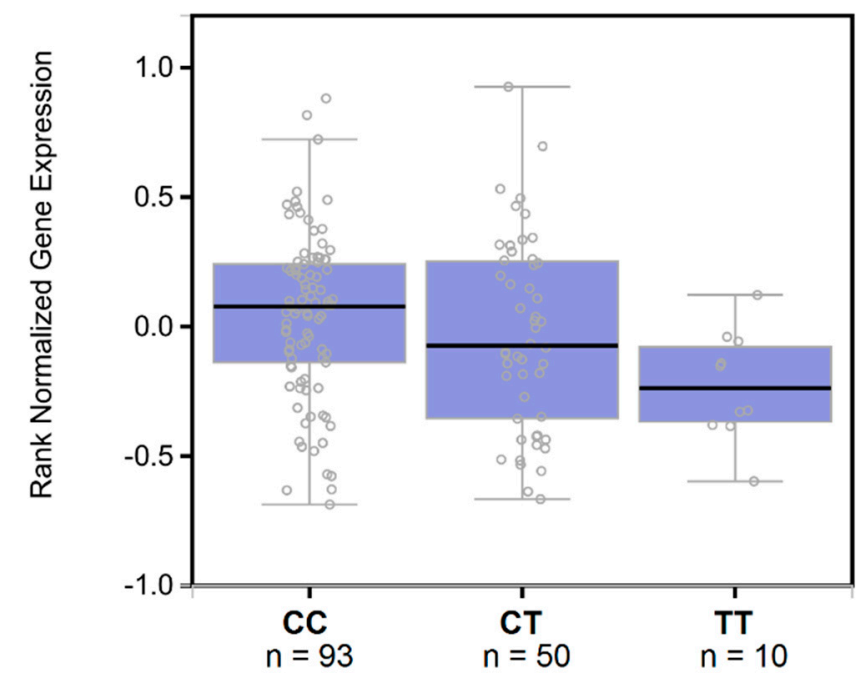

Figure 3. Expression quantitative trait loci box plots of associations between rs 9957088 genotypes and hepatic TTR gene expression (data source: GTEx V7). The purple box plot shows ranked normalized gene expression in median, 1st and 3rd quartiles, and 1.5 interquartile range (IQR) of 1st and 3rd quartiles.

Finally, we tested whether the effect of non-coding variation is affected by the haplotype phase of the Val122Ile mutation (cis-non-coding regulatory haplotype located on the same copy of the chromosome of Ile122 allele-versus trans-non-coding regulatory haplotype and Ile122 alleles located on different copies of the chromosome). The analysis was conducted considering the haplotype with the strongest functional evidence. We observed that the haplotype phase of the non-coding variation 
with respect to Val122Ile mutation did not affect the association observed (Val122 allele, $p=0.001$; Ile122 allele, $p=4 \times 10^{-4}$, Table 3).

Table 3. Associations with "having 10 or more outpatient surgeries" considering the haplotypic phase of non-coding regulatory haplotype (linkage disequilibrium (LD) Block \#16) and Val122Ile mutation in the carriers-only sample $(n=152)$.

\begin{tabular}{ccccc}
\hline Block \#16 Haplotype & $\begin{array}{c}\text { V122I } \\
(\mathbf{G}>\mathbf{A})\end{array}$ & $\begin{array}{c}\text { Case-Control } \\
\text { Frequencies }\end{array}$ & Chi Square & $\boldsymbol{p}$ Value \\
\hline CTTGTTCATACAGGCTATT & $\mathrm{A}$ & $0.286,0.417$ & 0.551 & 0.4581 \\
CTTGTTCATACAGGCTATT & $\mathrm{G}$ & $0.214,0.300$ & 0.275 & 0.5998 \\
TAAACCACCGTACATCTGC & $\mathrm{G}$ & $0.000,0.075$ & 0.646 & 0.4215 \\
TAAACCACCGTGCATCTGC & $\mathrm{G}$ & $0.000,0.066$ & 0.568 & 0.4509 \\
TTTGTCCCTACAGGCCTTT & $\mathrm{A}$ & $0.339,0.048$ & 12.482 & $4 \times 10^{-4}$ \\
TAAACCACCGTACATCTG & $\mathrm{A}$ & $0.000,0.033$ & 0.276 & 0.5996 \\
TTTGTCCCTACAGGCCTTT & $\mathrm{G}$ & $0.161,0.013$ & 10.55 & 0.0012 \\
CTTGTTCATACAGGCCTTT & $\mathrm{G}$ & $0.000,0.017$ & 0.137 & 0.7117 \\
\hline
\end{tabular}

Since no further information was available in the assessment of the Yale-Penn cohort regarding the types of outpatient surgeries, we investigated the association of the Val122Ile mutation with traits related to osteoarthritis in an independent cohort. These are recognized as early signs of the disease [31], and arthroplasty is a common procedure in outpatient settings [32]. This analysis was conducted using data of British participants from the UK Biobank. High-quality genotypic information is available for Val122Ile in the UK Biobank cohort (i.e., the variant is genotyped directly in the array used). Details regarding the data used in our analysis are available at [33]. Although there is a consistent difference in the carrier frequency of Val122Ile between our African-American cohort (3.5\%) and the UK Biobank cohort $(0.004 \%)$, the same clinical phenotype is present in Val122Ile carriers of European descent [34]. Accordingly, we observed a strong association of Val122Ile with the trait ICD10 M13: "Other disorders of the musculoskeletal system and connective tissue" $\left(n=361,194\right.$, beta $\left.=0.073, p=2.47 \times 10^{-13}\right)$. Additionally, when the analysis was stratified by sex, we observed the same male-specific associations observed in the African-American Val122Ile carriers $\left(n_{\text {male }}=167,020\right.$, beta $_{\text {male }}=0.14, p_{\text {male }}=4.02 \times$ $10^{-24}, \mathrm{n}_{\text {female }}=194,174$, beta $_{\text {female }}=-0.002, p_{\text {female }}=0.921$ ).

\section{Discussion}

TTR amyloidosis is a rare life-threatening disease with complex phenotype-genotype correlations characterized by strong variability in penetrance, age of onset, and clinical symptoms $[35,36]$. Several studies have been conducted to understand the mechanisms determining the phenotypic variability observed among the carriers of TTR amyloidogenic mutations [37-40]. However, although Val30Met and Val122Ile are the most frequent TTR mutations, there is an evident disparity in the ongoing research efforts to understand the molecular mechanisms associated with these disease-causing variants. This is in line with the well-known population disparity in human genetic research [41]. Additionally, the generally lower socioeconomic status among the AA population compared to European Americans is a possible explanation for the general lack of representation of TTR Val122Ile carriers among the cohorts of patients with hereditary cardiac amyloidosis [4]. The present study aimed to reduce the population disparity in TTR amyloidosis research, investigating two aspects of TTR Val122Ile pathogenicity: (i) The early phenotypic manifestation of the mutation in AA carriers; (ii) Whether non-coding variation in regulatory elements modulates the health status of AA carriers.

The association analysis of Val122Ile with medical history identified a significant result for carriers having 10 or more outpatient surgeries. In the SSADDA assessment, outpatient surgery was defined as surgery that did not require an overnight hospital stay. Having 10 or more outpatient surgeries is an extreme phenotype that occurred in a limited number of subjects. According to our permutation 
analysis $(n=10,000)$, the most likely random scenario is that no Val122Ile carrier would be a case for this phenotypic trait.

Unfortunately, no further information is available regarding the type of outpatient surgeries undergone by the Yale-Penn participants. Although the main phenotypic expression of Val122Ile mutation is cardiac, multiorgan involvement was also observed among carriers, with gait, gastrointestinal, neurological, urinary/renal, and ocular symptoms also reported [36,42]. Since the age of the Yale-Penn cohort is relatively lower than the age of onset observed in patients with the TTR Val122Ile mutation, we hypothesize that our observed association was due to an early expression of the disease. Arthroplasty, an increasingly common procedure in outpatient settings [32], has been reported as an early sign of transthyretin cardiac amyloidosis (i.e., the main phenotype associated with TTR Val122Ile mutation), occurring approximately seven years before the disease diagnosis [43]. This symptom is due to the TTR amyloid deposition in the articular cartilage, contributing to the cell and extracellular matrix damage observed in osteoarthritic joints [31]. To test the hypothesis that our result regarding outpatient surgeries is related to the early occurrence of arthroplasty, we investigated the association of Val122Ile with musculoskeletal and connective-tissue disorders observed in a large independent cohort of European descent. Notwithstanding the different ancestral background, a similar clinical phenotype is expected in Val122Ile carriers of African and European descent [34]. Accordingly, we observed a strong association with musculoskeletal and connective-tissue disorders that follows the same male-specific pattern observed in the African-American cohort. Although this result supports our hypothesis, further studies are needed to confirm that outpatient surgeries in Val122Ile carriers are related to the TTR amyloid deposition in the articular cartilage.

Because of the difficulty in recognizing symptoms outside of the context of a specialized diagnostic environment, the diagnosis of TTR amyloidosis frequently occurs several years after the emergence of the first signs, with clinical procedures performed to help to alleviate the symptoms [44] (if the diagnosis is ever even made at all). Under this scenario, the association between Val122Ile and outpatient surgeries in our relatively young cohort indicates that, in certain carriers, the very early symptoms of the disease may be multiorgan rather than organ-specific. However, our data also confirmed the relationship between Val122Ile and cardiac function. We observed an effect size in the association between the TTR mutation and heart disease, similar to the one reported by a previous analysis of a general-population cohort [45]. Additionally, there is a relationship between having 10 or more outpatient surgeries and heart disease among the Val122Ile carriers investigated that is not present among the non-carriers. This suggests that the early signs-like the musculoskeletal and connective-tissue disorders observed in UK Biobank-causing multiple clinical procedures are linked to the cardiac symptoms.

Previous studies indicated that non-coding variation in regulatory elements is involved in the phenotypic heterogeneity observed among carriers of TTR mutations where penetrance, age of onset, and organ involvement are likely modulated by changes in transcriptomic regulation $[13,14]$. However, no previous investigation was conducted to test this hypothesis among AA carriers of TTR Val122Ile. In our study, we observed that, among Val122Ile carriers, genetic variability upstream of the TTR gene is associated with the phenotype identified in the carriers-versus-non-carriers analysis. In silico analyses confirmed that this variation is located in predicted functional elements that regulate TTR gene expression in the liver, the main organ source of the amyloidogenic protein. The association of Val122Ile with "having 10 or more outpatient surgeries" was not age-dependent. This suggests that certain Val122Ile carriers with a specific non-coding regulatory haplotype may show the first signs of the disease earlier than Val122Ile carriers without the regulatory non-coding haplotype. Additionally, the same TTR non-coding haplotypes are also associated with changes of haptoglobin protein in the blood. This result is particularly interesting since haptoglobin is one of the extracellular chaperones overrepresented in transthyretin amyloidosis [46]. Accordingly, we hypothesize that the non-coding regulatory haplotypes affect both transcriptomic and proteomic regulation of the TTR gene, causing the activation of extracellular chaperones to cope with amyloid-prone proteins. 


\section{Conclusions}

We report novel data regarding how Val122Ile affects the health status of carriers of African descent at an earlier point in life than the expected late-onset disease. Additionally, we provide the first evidence of the role of non-coding variability and gene expression regulation in the phenotypic presentation among AA carriers, similar to what was observed in other studies for other TTR mutations. These data start to explain the clinical variability observed in subjects who are carriers of the disease-causing mutation. Further studies based on a deeper phenotypic characterization will be needed to explore the hypotheses generated by the present investigation. The high frequency of Val122Ile in AAs permits the investigation of a large number of individuals, providing a tool for understanding the molecular mechanisms and epidemiological associations present in TTR amyloidosis. Unlike Val122Ile allele frequency in individuals of African descent (1.5\%), TTR amyloidogenic mutations have a lower allele frequency $(<0.3 \%)$; therefore, a much larger sample size will be needed to investigate their phenotypic spectrum.

Supplementary Materials: The following are available online at http:/ /www.mdpi.com/2077-0383/8/2/269/ s1, Figure S1: Genomic region investigated in the haplotype association analysis (GRCh37/hg19 chr 18: $28,171,770-30,174,635)$. The regions highlighted in light blue indicate the non-coding haplotypes identified as significant. TTR gene region is highlighted in red. Table S1: Variants included in the haplotype association analysis. Information regarding the imputation quality (Info score) in Illumina HumanCoreExome array (HCE) and Illumina HumanOmni1-Quad v1.0 microarray (OMNI) is also reported. Table S2: Details of the association analysis between Val122Ile and traits related to medical history. Beta values are reported as effect size for linear regression models. Odds Ratios are reported as effect size for logistic regression models. Table S3: LD blocks identified in the genomic regions investigated in the haplotype association analysis (GRCh37/hg19 chr 18: $28,171,770-30,174,635)$. The rsId of the variants are reported in Table S1. Table S4: Association results of all haplotypes tested with respect to "having 10 or more outpatient surgeries" among Val122Ile carriers. Table S5: Functional annotation (Haploreg v4.1) of non-coding haplotypes identified as significantly associated with "having 10 or more outpatient surgeries" among Val122Ile carriers.

Author Contributions: Conceptualization, R.P.; Methodology, R.P.; Investigation, R.P., Y.Z.N, and J.G.; Writing-Original Draft Preparation, R.P.; Writing-Review \& Editing, Y.Z.N, and J.G.; Funding Acquisition, J.G.

Funding: The Yale-Penn cohort was funded by grants from the US National Institutes of Health (RC2 DA028909, R01 DA12690, R01 DA12849, R01 DA18432, R01AA11330, and R01 AA017535).

Acknowledgments: We thank Henry R. Kranzler of the University of Pennsylvania (Philadelphia, PA, USA) for co-leading the recruitment of the Yale-Penn cohort.

Conflicts of Interest: R.P. received a research grant from Pfizer (Global ASPIRE) to investigate epigenetic changes in African-American carriers of TTR V122I mutation. The other authors declare that they have no competing financial interests.

\section{References}

1. Plante-Bordeneuve, V.; Said, G. Familial amyloid polyneuropathy. Lancet Neurol. 2011, 10, $1086-1097$. [CrossRef]

2. Ruberg, F.L.; Berk, J.L. Transthyretin (ttr) cardiac amyloidosis. Circulation 2012, 126, 1286-1300. [CrossRef] [PubMed]

3. Parman, Y.; Adams, D.; Obici, L.; Galan, L.; Guergueltcheva, V.; Suhr, O.B.; Coelho, T. European Network for TTR-FAP (ATTReuNET). Sixty years of transthyretin familial amyloid polyneuropathy (ttr-fap) in europe: Where are we now? A european network approach to defining the epidemiology and management patterns for ttr-fap. Curr. Opin. Neurol. 2016, 29 (Suppl. 1), S3-S13. [CrossRef] [PubMed]

4. Buxbaum, J.N.; Ruberg, F.L. Transthyretin v122i (pv142i)* cardiac amyloidosis: An age-dependent autosomal dominant cardiomyopathy too common to be overlooked as a cause of significant heart disease in elderly african americans. Genet. Med. 2017, 19, 733-742. [CrossRef] [PubMed]

5. Araki, S.; Ando, Y. Transthyretin-related familial amyloidotic polyneuropathy-progress in kumamoto, japan (1967-2010). Proc. Jpn. Acad. Ser. B Phys. Biol. Sci. 2010, 86, 694-706. [CrossRef] [PubMed]

6. Jacobson, D.R.; Alexander, A.A.; Tagoe, C.; Buxbaum, J.N. Prevalence of the amyloidogenic transthyretin (ttr) v122i allele in 14333 african-americans. Amyloid 2015, 22, 171-174. [CrossRef] [PubMed] 
7. Jacobson, D.R.; Alexander, A.A.; Tagoe, C.; Garvey, W.T.; Williams, S.M.; Tishkoff, S.; Modiano, D.; Sirima, S.B.; Kalidi, I.; Toure, A.; et al. The prevalence and distribution of the amyloidogenic transthyretin (ttr) v122i allele in africa. Mol. Genet. Genomic Med. 2016, 4, 548-556. [CrossRef]

8. Reddi, H.V.; Jenkins, S.; Theis, J.; Thomas, B.C.; Connors, L.H.; Van Rhee, F.; Highsmith, W.E., Jr. Homozygosity for the v122i mutation in transthyretin is associated with earlier onset of cardiac amyloidosis in the african american population in the seventh decade of life. J. Mol. Diagn. 2014, 16, 68-74. [CrossRef]

9. Alves-Ferreira, M.; Coelho, T.; Santos, D.; Sequeiros, J.; Alonso, I.; Sousa, A.; Lemos, C. A trans-acting factor may modify age at onset in familial amyloid polyneuropathy attrv30m in portugal. Mol. Neurobiol. 2018, 55, 3676-3683. [CrossRef]

10. Polimanti, R.; Di Girolamo, M.; Manfellotto, D.; Fuciarelli, M. In silico analysis of ttr gene (coding and non-coding regions, and interactive network) and its implications in transthyretin-related amyloidosis. Amyloid 2014, 21, 154-162. [CrossRef]

11. Iorio, A.; De Angelis, F.; Di Girolamo, M.; Luigetti, M.; Pradotto, L.; Mauro, A.; Manfellotto, D.; Fuciarelli, M.; Polimanti, R. Most recent common ancestor of $\operatorname{ttr}$ val30met mutation in italian population and its potential role in genotype-phenotype correlation. Amyloid 2015, 22, 73-78. [CrossRef] [PubMed]

12. Polimanti, R.; Di Girolamo, M.; Manfellotto, D.; Fuciarelli, M. Functional variation of the transthyretin gene among human populations and its correlation with amyloidosis phenotypes. Amyloid 2013, 20, 256-262. [CrossRef] [PubMed]

13. Iorio, A.; De Angelis, F.; Di Girolamo, M.; Luigetti, M.; Pradotto, L.G.; Mazzeo, A.; Frusconi, S.; My, F.; Manfellotto, D.; Fuciarelli, M.; et al. Population diversity of the genetically determined ttr expression in human tissues and its implications in ttr amyloidosis. BMC Genom. 2017, 18, 254. [CrossRef] [PubMed]

14. Iorio, A.; De Lillo, A.; De Angelis, F.; Di Girolamo, M.; Luigetti, M.; Sabatelli, M.; Pradotto, L.; Mauro, A.; Mazzeo, A.; Stancanelli, C.; et al. Non-coding variants contribute to the clinical heterogeneity of ttr amyloidosis. Eur. J. Hum. Genet. 2017, 25, 1055-1060. [CrossRef] [PubMed]

15. Pierucci-Lagha, A.; Gelernter, J.; Feinn, R.; Cubells, J.F.; Pearson, D.; Pollastri, A.; Farrer, L.; Kranzler, H.R. Diagnostic reliability of the semi-structured assessment for drug dependence and alcoholism (ssadda). Drug Alcohol Depend. 2005, 80, 303-312. [CrossRef] [PubMed]

16. Pierucci-Lagha, A.; Gelernter, J.; Chan, G.; Arias, A.; Cubells, J.F.; Farrer, L.; Kranzler, H.R. Reliability of dsm-iv diagnostic criteria using the semi-structured assessment for drug dependence and alcoholism (ssadda). Drug Alcohol Depend. 2007, 91, 85-90. [CrossRef] [PubMed]

17. Polimanti, R.; Zhao, H.; Farrer, L.A.; Kranzler, H.R.; Gelernter, J. Ancestry-specific and sex-specific risk alleles identified in a genome-wide gene-by-alcohol dependence interaction study of risky sexual behaviors. Am. J. Med. Genet. Part B Neuropsychiatr. Genet. 2017, 174, 846-853. [CrossRef] [PubMed]

18. Polimanti, R.; Kaufman, J.; Zhao, H.; Kranzler, H.R.; Ursano, R.J.; Kessler, R.C.; Gelernter, J.; Stein, M.B. A genome-wide gene-by-trauma interaction study of alcohol misuse in two independent cohorts identifies prkg1 as a risk locus. Mol. Psychiatry 2018, 23, 154-160. [CrossRef] [PubMed]

19. Gelernter, J.; Kranzler, H.R.; Sherva, R.; Koesterer, R.; Almasy, L.; Zhao, H.; Farrer, L.A. Genome-wide association study of opioid dependence: Multiple associations mapped to calcium and potassium pathways. Biol. Psychiatry 2014, 76, 66-74. [CrossRef] [PubMed]

20. Polimanti, R.; Zhang, H.; Smith, A.H.; Zhao, H.; Farrer, L.A.; Kranzler, H.R.; Gelernter, J. Genome-wide association study of body mass index in subjects with alcohol dependence. Addict. Biol. 2017, 22, 535-549. [CrossRef] [PubMed]

21. Das, S.; Forer, L.; Schonherr, S.; Sidore, C.; Locke, A.E.; Kwong, A.; Vrieze, S.I.; Chew, E.Y.; Levy, S.; McGue, M.; et al. Next-generation genotype imputation service and methods. Nat. Genet. 2016, 48, 1284-1287. [CrossRef] [PubMed]

22. 1000 Genomes Project Consortium; Auton, A.; Brooks, L.D.; Durbin, R.M.; Garrison, E.P.; Kang, H.M.; Korbel, J.O.; Marchini, J.L.; McCarthy, S.; McVean, G.A.; et al. A global reference for human genetic variation. Nature 2015, 526, 68-74. [PubMed]

23. Chang, C.C.; Chow, C.C.; Tellier, L.C.; Vattikuti, S.; Purcell, S.M.; Lee, J.J. Second-generation plink: Rising to the challenge of larger and richer datasets. Gigascience 2015, 4, 7. [CrossRef] [PubMed]

24. Barrett, J.C. Haploview: Visualization and analysis of snp genotype data. Cold Spring Harb. Protoc. $2009,10$. [CrossRef] [PubMed] 
25. Gabriel, S.B.; Schaffner, S.F.; Nguyen, H.; Moore, J.M.; Roy, J.; Blumenstiel, B.; Higgins, J.; DeFelice, M.; Lochner, A.; Faggart, M.; et al. The structure of haplotype blocks in the human genome. Science 2002, 296, 2225-2229. [CrossRef] [PubMed]

26. Ward, L.D.; Kellis, M. Haploreg: A resource for exploring chromatin states, conservation, and regulatory motif alterations within sets of genetically linked variants. Nucleic Acids Res. 2012, 40, D930-D934. [CrossRef] [PubMed]

27. Boyle, A.P.; Hong, E.L.; Hariharan, M.; Cheng, Y.; Schaub, M.A.; Kasowski, M.; Karczewski, K.J.; Park, J.; Hitz, B.C.; Weng, S.; et al. Annotation of functional variation in personal genomes using regulomedb. Genome. Res. 2012, 22, 1790-1797. [CrossRef] [PubMed]

28. GTEx Consortium. Human genomics. The genotype-tissue expression (gtex) pilot analysis: Multitissue gene regulation in humans. Science 2015, 348, 648-660. [CrossRef]

29. Jacobson, D.; Tagoe, C.; Schwartzbard, A.; Shah, A.; Koziol, J.; Buxbaum, J. Relation of clinical, echocardiographic and electrocardiographic features of cardiac amyloidosis to the presence of the transthyretin v122i allele in older african-american men. Am. J. Cardiol. 2011, 108, 440-444. [CrossRef]

30. Sun, B.B.; Maranville, J.C.; Peters, J.E.; Stacey, D.; Staley, J.R.; Blackshaw, J.; Burgess, S.; Jiang, T.; Paige, E.; Surendran, P.; et al. Genomic atlas of the human plasma proteome. Nature 2018, 558, 73-79. [CrossRef]

31. Akasaki, Y.; Reixach, N.; Matsuzaki, T.; Alvarez-Garcia, O.; Olmer, M.; Iwamoto, Y.; Buxbaum, J.N.; Lotz, M.K. Transthyretin deposition in articular cartilage: A novel mechanism in the pathogenesis of osteoarthritis. Arthritis Rheumatol. 2015, 67, 2097-2107. [CrossRef] [PubMed]

32. Bert, J.M.; Hooper, J.; Moen, S. Outpatient total joint arthroplasty. Curr. Rev. Musculoskelet Med. 2017, 10, 567-574. [CrossRef] [PubMed]

33. UK Biobank GWAS. Available online: https://github.com/Nealelab/UK_Biobank_GWAS (accessed on 2 February 2019).

34. Cappelli, F.; Frusconi, S.; Bergesio, F.; Grifoni, E.; Fabbri, A.; Giuliani, C.; Falconi, S.; Bonifacio, S.; Perfetto, F. The val142ile transthyretin cardiac amyloidosis: Not only an afro-american pathogenic variant? A single-centre italian experience. J. Cardiovasc Med. (Hagerstown) 2016, 17, 122-125. [CrossRef] [PubMed]

35. Coelho, T.; Maurer, M.S.; Suhr, O.B. Thaos-the transthyretin amyloidosis outcomes survey: Initial report on clinical manifestations in patients with hereditary and wild-type transthyretin amyloidosis. Curr. Med. Res. Opin. 2013, 29, 63-76. [CrossRef] [PubMed]

36. Maurer, M.S.; Hanna, M.; Grogan, M.; Dispenzieri, A.; Witteles, R.; Drachman, B.; Judge, D.P.; Lenihan, D.J.; Gottlieb, S.S.; Shah, S.J.; et al. Genotype and phenotype of transthyretin cardiac amyloidosis: Thaos (transthyretin amyloid outcome survey). J. Am. Coll. Cardiol. 2016, 68, 161-172. [CrossRef] [PubMed]

37. Santos, D.; Santos, M.J.; Alves-Ferreira, M.; Coelho, T.; Sequeiros, J.; Alonso, I.; Oliveira, P.; Sousa, A.; Lemos, C.; Grazina, M. Mtdna copy number associated with age of onset in familial amyloid polyneuropathy. J. Neurol. Neurosurg Psychiatry 2018, 89, 300-304. [CrossRef] [PubMed]

38. Panayiotou, E.; Papacharalambous, R.; Antoniou, A.; Christophides, G.; Papageorgiou, L.; Fella, E.; Malas, S.; Kyriakides, T. Genetic background modifies amyloidosis in a mouse model of attr neuropathy. Biochem. Biophys. Rep. 2016, 8, 48-54. [CrossRef]

39. Chan, G.G.; Koch, C.M.; Connors, L.H. Serum proteomic variability associated with clinical phenotype in familial transthyretin amyloidosis (attrm). J. Proteome Res. 2017, 16, 4104-4112. [CrossRef]

40. Moreira, L.; Beirao, J.M.; Beirao, I.; Pinho e Costa, P. Oligomeric ttr v30m aggregates compromise cell viability, erythropoietin gene expression and promoter activity in the human hepatoma cell line hep3b. Amyloid 2015, 22, 93-99. [CrossRef]

41. Haga, S.B. Impact of limited population diversity of genome-wide association studies. Genet. Med. 2010, 12, 81-84. [CrossRef]

42. Carr, A.S.; Pelayo-Negro, A.L.; Jaunmuktane, Z.; Scalco, R.S.; Hutt, D.; Evans, M.R.; Heally, E.; Brandner, S.; Holton, J.; Blake, J.; et al. Transthyretin v122i amyloidosis with clinical and histological evidence of amyloid neuropathy and myopathy. Neuromuscul. Disord. 2015, 25, 511-515. [CrossRef] [PubMed]

43. Rubin, J.; Alvarez, J.; Teruya, S.; Castano, A.; Lehman, R.A.; Weidenbaum, M.; Geller, J.A.; Helmke, S.; Maurer, M.S. Hip and knee arthroplasty are common among patients with transthyretin cardiac amyloidosis, occurring years before cardiac amyloid diagnosis: Can we identify affected patients earlier? Amyloid 2017, 24, 226-230. [CrossRef] [PubMed] 
44. Adams, D.; Suhr, O.B.; Hund, E.; Obici, L.; Tournev, I.; Campistol, J.M.; Slama, M.S.; Hazenberg, B.P.; Coelho, T. European Network for TTR-FAP (ATTReuNET). First european consensus for diagnosis, management, and treatment of transthyretin familial amyloid polyneuropathy. Curr. Opin. Neurol. 2016, 29 (Suppl. 1), S14-S26. [CrossRef] [PubMed]

45. Quarta, C.C.; Buxbaum, J.N.; Shah, A.M.; Falk, R.H.; Claggett, B.; Kitzman, D.W.; Mosley, T.H.; Butler, K.R.; Boerwinkle, E.; Solomon, S.D. The amyloidogenic v122i transthyretin variant in elderly black americans. New Engl. J. Med. 2015, 372, 21-29. [CrossRef] [PubMed]

46. Da Costa, G.; Ribeiro-Silva, C.; Ribeiro, R.; Gilberto, S.; Gomes, R.A.; Ferreira, A.; Mateus, E.; Barroso, E.; Coelho, A.V.; Freire, A.P.; et al. Transthyretin amyloidosis: Chaperone concentration changes and increased proteolysis in the pathway to disease. PLOS ONE 2015, 10, e0125392. [CrossRef]

2019 by the authors. Licensee MDPI, Basel, Switzerland. This article is an open access article distributed under the terms and conditions of the Creative Commons Attribution (CC BY) license (http:/ / creativecommons.org/licenses/by/4.0/). 